\section{Airborne microbial flora in buffalo farms in a Mediterranean climate}

\author{
Debora Stelitano, ${ }^{1}$ Giuseppe Squillaci, ${ }^{2}$ \\ Biagio Santella, ${ }^{1}$ Annalisa Chianese, ${ }^{1}$ \\ Virginia D'Oriano, ${ }^{1}$ \\ Emiliana Finamore, ${ }^{1}$ \\ Mariateresa Vitiello, ${ }^{3}$ \\ Federica Maria di Lella, ${ }^{1}$ \\ Marilena Galdiero, ${ }^{1}$ Gianluigi Franci ${ }^{1}$ \\ ${ }^{1}$ Department of Experimental Medicine, \\ University of Campania Luigi Vanvitelli, \\ Naples; ${ }^{2}$ Research Institute on \\ Terrestrial Ecosystems, Italian National \\ Research Council (CNR), Naples; \\ ${ }^{3}$ Department of Clinical Pathology, \\ Virology Unit, San Giovanni di Dio e \\ Ruggi d'Aragona Hospital, Salerno, \\ Italy
}

\begin{abstract}
The last few decades have seen an increase in intensive buffalo farms worldwide. Such industrialized activity entails human and animal potential health-related hazards as well as for the environment. Given the breadth of the issue, in this study we focused on gathering microbiological air sampling in the Southern Italian buffalo farms in order to obtain further information on airborne microbial flora. In details, we evaluated the bio-aerosol concentration of cultivable bacteria and fungi in ten different buffalo farms. There are evidences showing that exposure to organic dust may exacerbate asthma, just as it may cause mucous membrane irritation and chronic bronchitis. Likewise, studies show that inhaling noninfectious microorganisms and their components may cause inflammation of the respiratory tract. As a result, this is a significant health hazard to these farms' workers as well as to rural residents living closely to them.
\end{abstract}

\section{Introduction}

In Italy, approximately 200,000 Mediterranean water buffaloes (Bubalus bubalis) are kept, mainly in the central and southern regions, where they constitute an important part of the animal industry in terms of milk-derived products. ${ }^{1}$ The water buffalo is a valuable species since it is considered a multipurpose animal with several valuable features such as its meat, horns and skin. ${ }^{2}$ Moreover, its rich and nutritious milk is highly precious, and can be converted into many kinds of cheese, primarily mozzarella, which is one of the major economical reality of Southern Italy. Modern agricultural methods have changed the way buffalo are raised. ${ }^{3,4}$ To increase milk production and in order to reduce farm-labour, the previous methodology based on allowing animal to graze in the fields has changed and animals are now stocked in confinement buildings. Buffaloes are managed within different types of confinement sectors according to their growth stage and operational process. The stages of buffalos breeding can be categorized as: i) lactating offspring, ii) nursery, iii) growing, iv) milking. ${ }^{5}$

Microorganisms and their components or products, deriving from organic waste (feces and feed), are easily cumulated and aerosolized in stables with high animal population density. ${ }^{6,7}$ Inhalation of airborne microorganisms is considered an important aspect in the prevalence of respiratory diseases within the farming community. ${ }^{8,9}$ One major problem is the inhalation of airborne gram-negative bacteria and allergenic fungi..$^{5,10}$ Working in animal housings may be correlated with more frequent exposure to organic dust, microorganisms and bacterial components present in animal wastes in amounts which once inhaled are able to cause respiratory diseases following induction of cellular and immunological responses. ${ }^{11-13}$ The species composition and concentration of airborne microorganisms can be highly affected by environmental factors such as climate and humidity, but an important role is also played by the farm characteristics, such as feeding and cleaning practices. ${ }^{14-16}$ To the best of our knowledge, no information regarding the level of airborne microorganisms in buffalo farm has been previously reported. The major aim of this study was to investigate the concentrations of airborne microorganisms and to examine the species composition in a vast number of buffalo stables located in Southern Italy. ${ }^{17}$

\section{Materials and Methods}

\section{Study site}

Ten buffalo farms were selected. The buffalo farms were located in Campania, a region of Southern Italy with a Mediterranean climate. Selected farms were homogeneous in size and organization with growing house and milking parlour naturally ventilated, and open-air house. Each growing house has the capacity to hold 400 buffaloes.
Correspondence: Gianluigi Franci, Department of Experimental Medicine, University of Campania Luigi Vanvitelli, Via Costantinopoli 16, 80138 Naples, Italy.

E-mail: gianluigi.franci@unicampania.it

Key words: Buffalo; Microorganism; Farms; Milk.

Contributions: DS data collecting and manuscript reviewing; GS, BS, AC, VD, EF, MV, FMDL, MG manuscript reviewing and reference search; GF data collecting, figures preparation and manuscript writing.

Conflict of interest: the authors declare no potential conflict of interest.

Funding: VALEREplus Program.

Received for publication: 1 March 2019.

Revision received: 17 March 2019.

Accepted for publication: 18 March 2019.

This work is licensed under a Creative Commons Attribution 4.0 License (by-nc 4.0).

CCopyright D. Stelitano et al., 2019

Licensee PAGEPress, Italy

Translational Medicine Reports 2019; 3:8146 doi:10.4081/tmr.8146

\section{Collection of air samples}

The bioaerosol samples were collected with a Thermo-Scientific six-stage Andersen Cascade Impactor, following the method described by Andersen et al. ${ }^{3}$ The Andersen sampler operated for $1 \mathrm{~min}$ to obtain the total number of airborne aerobic bacteria and for $20 \mathrm{~min}$ to obtain the number of airborne Gram-negative and Grampositive bacteria at an air-flow rate of 28.3 $1 / \mathrm{min}$. Samples of airborne culturable bacteria, Gram-negative bacteria, and culturable fungi were simultaneously collected $1.5 \mathrm{~m}$ above the floor of the central walkway in each stable of the examined buffalo farms. In particular, $10 \mathrm{~L}$ of air were collected in the stables and $50 \mathrm{~L}$ of air were collected in the parlours before milking (when the rooms had been cleaned) and after milking (once all animals have left the parlour and no cleaning has been performed yet). For each farm, a series of three samples taken from five different locations within each stable were plated of the following media: Rose Bengal Agar (RBA), Malt Extract Agar (MEA), Mac Conkey medium (MAC), Plate Agar Count or Sabouraud dextrose agar. RBA (without streptomycin) was prepared using Martin's modification of peptone dextrose agar. MEA was prepared by using $25 \mathrm{~g}$ of malt extract per litre, $1 \mathrm{~g}$ of yeast extract per liter, and $16.5 \mathrm{~g}$ of agar per liter. 
The grown colonies were counted on all plates, and counts from dilution plates containing 30-300 colony-forming units (CFUs) were used in back-calculations to determine the concentration of isolated bacteria per cubic meter of air within each buffalo stable.

\section{Sample analysis}

We analyzed bacterial and fungal air samples collected with bioaerosol samplers as described before. The temperature and relative humidity were monitored throughout the sampling periods and were $22 \pm 1^{\circ} \mathrm{C}$ and $26 \pm 4 \%$ respectively. After sampling, the plates were immediately incubated at $30^{\circ} \mathrm{C}$ for 24 to $36 \mathrm{~h}$ for mesophilic bacteria, and at $37^{\circ} \mathrm{C}$ for $24 \mathrm{~h}$ to $36 \mathrm{~h}$ for coliforms. For yeasts and moulds the cultures were incubated at room temperature $\left(23 \pm 3^{\circ} \mathrm{C}\right)$. The plates were inspected after 4 days and periodically up to 14 days after primary exposure. The concentration of bacteria and fungi per cubic meter of air were calculated, and the most common genera of aerobic bacteria present were identified by Gram staining and biochemical methods using bacterial identification kits (Becton Dickinson Micro System), while the fungi present were identified by examination of colony morphology, followed by microscopic examination of spores and hyphae. ${ }^{18}$

However, for the great majority of samples all bacterial and fungal types present were identified and their concentration was expressed as $\mathrm{CFU} / \mathrm{m}^{3}$ of air. Actual plate counts, not estimated counts, was used to calculate the $\mathrm{CFU} / \mathrm{m}^{3}$. Most fungi were identified to the genus level according to published references. ${ }^{19}$

\section{Results}

The concentration of bacteria and fungi detected in the farms was determined by using optimal sampling methods in a way that would properly minimize colony masking bioaereosol. The results showed the concentrations of cultivable microorganisms in 10 farms in Southern Italy during the year 2018 and relative to the four seasons: winter, spring, summer and fall.

The concentration of cultured bacteria in the air was significantly high in stables. In Table 1 we report the minimum and maximum values of the bacterial concentration found, ranging from $7.9 \times 10^{4}$ to $2.4 \times 10^{6}$ $\mathrm{CFU} / \mathrm{m}^{3}$ during the summer, $6.5 \times 10^{4}$ to $1.1 \times 10^{6} \mathrm{CFU} / \mathrm{m}^{3}$ during the spring, $6.2 \times 10^{4}$ to $9.8 \times 10^{5} \mathrm{CFU} / \mathrm{m}^{3}$ during the fall and $6.3 \times 10^{3}$ to $3.2 \times 10^{5} \mathrm{CFU} / \mathrm{m}^{3}$ during the winter. Likewise, the values for fungi are $9.1 \times 10^{2}$ to $4.1 \times 10^{3} \mathrm{CFU} / \mathrm{m}^{3}$ during the sum- mer, $6.6 \times 10^{2} \mathrm{CFU} / \mathrm{m}^{3}$ to $3.3 \times 10^{3}$ during the spring, $7.1 \times 10^{2}$ to $2.9 \times 10^{3} \mathrm{CFU} / \mathrm{m}^{3}$ during the fall and $3.3 \times 10^{2}$ to $1.7 \times 10^{3} \mathrm{CFU} / \mathrm{m}^{3}$ during the winter (Table 1).

For an immediate understanding the results are shown in a histogram in Figure 1. Analysis of variance showed no significant difference in airborne fungi concentration among buffalo stables $(\mathrm{P}=0.25)$.

However, a great variety of bacterial and fungal species or genera were found in the buffalo farms. Moreover, the bacteria and the fungi identified in the stables were characterized and the results are consistent with the published literature on the subject.

In Figure 2 we represented, with a histogram, the families of gram negative bacteria found in breeding farms. Among these, the most numerous belonged to the following species: Enterobacteriaceae, Campylobacteriacae and Pseudomonadaceae. Within the Enterobacteriaceae, Escherichia coli and Pantoea agglomerans were predominating species. Regarding the Pseudomonadaceae, the presence of Pseudomonas aeruginosa was correlated with relative humidity of air.
Moreover, the high percentage of Pseudomonadaceae in milking parlours compared to stables could depend on the air humidity following cleaning of milking parlours by water spraying performed routinely. No obligate anaerobic Gram-negative bacteria were isolated. The main airborne concentrations of Gram-positive bacteria were relatively high in the same stables and among them the predominant genera identified were Bacillus, Enterococcus, Corynebacterium and Clostridium (Figure 3). Significantly higher concentrations of airborne fungi were measured in breading houses. Candida, Saccharomyces, Aspergillus and Cladosporium were the most representative of the identified fungi in all stables. The other species of fungi found were Alternaria, Penicillium, and Fusarium (Figure 4).

Low levels of cultivable fungi and bacteria were determined in the air of the milking parlours, especially during the stage before milking. In milking parlours, during the stage before milking the microbial concentrations ranged from $7.1 \times 10^{2}$ to $2.9 \times 10^{3}$ $\mathrm{CFU} / \mathrm{m}^{3}$ during the spring, $9.1 \times 10^{2}$ to

\section{BACTERIA IN ALL STABLES}
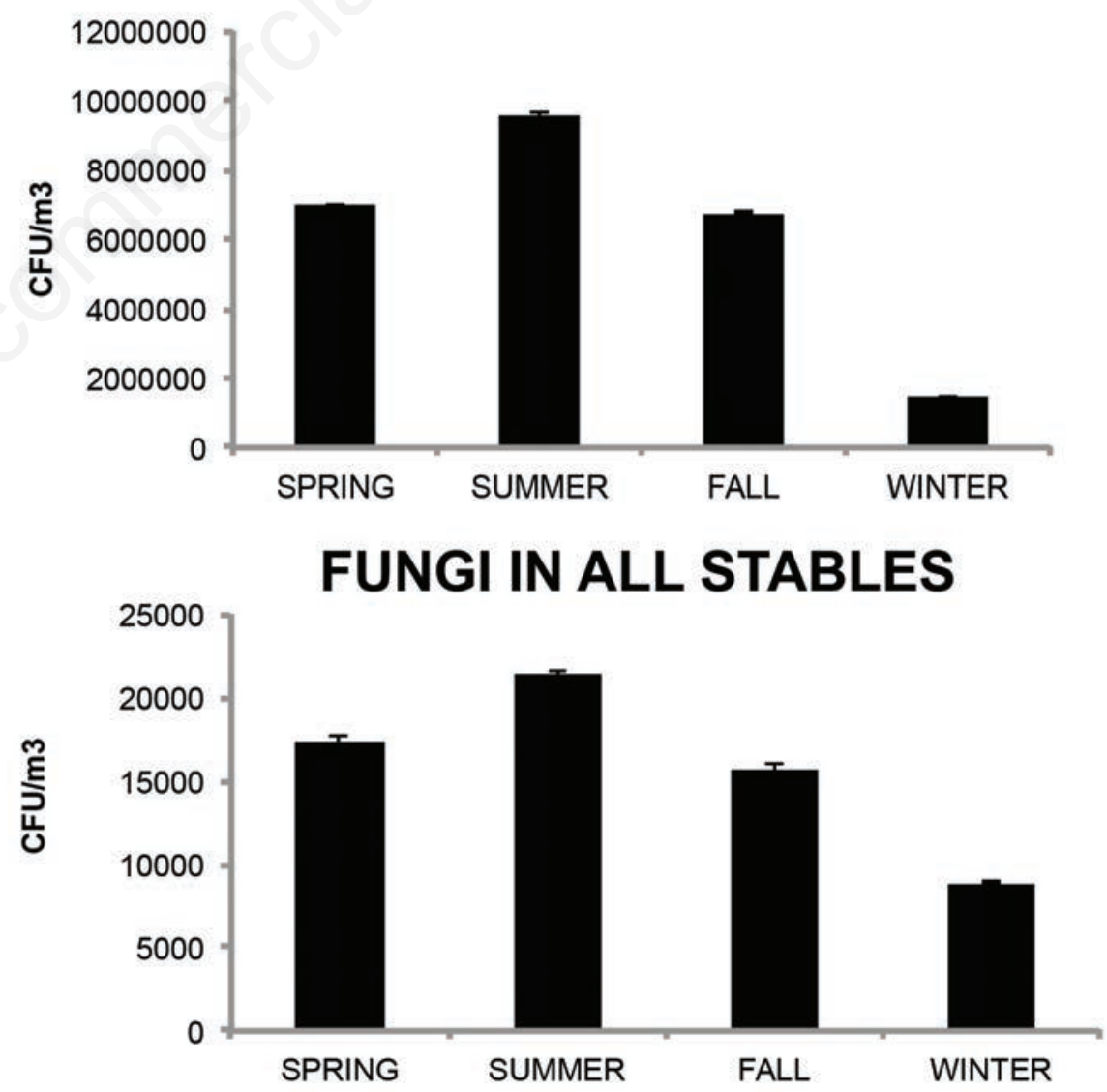

Figure 1. Concentration of bacteria and fungi in all the stable of all farms analyzed during the four season of the year 2018. CFU, colony-forming unit. 
$4.4 \times 10^{3} \mathrm{CFU} / \mathrm{m}^{3}$ during the summer, $2.3 \times 10^{2}$ to $1.1 \times 10^{3} \mathrm{CFU} / \mathrm{m}^{3}$ during the $6.1 \times 10^{2}$ to $3.2 \times 10^{3} \mathrm{CFU} / \mathrm{m}^{3}$ during the fall spring, $3.1 \times 10^{2} \mathrm{CFU} / \mathrm{m}^{3}$ to $2.2 \times 10^{3}$ during and $2.3 \times 10^{2}$ to $1.9 \times 10^{3} \mathrm{CFU} / \mathrm{m}^{3}$ during the the summer, from $2.6 \times 10^{2}$ to $9.9 \times 10^{2}$ winter for bacteria (Table 2) and from

$\mathrm{CFU} / \mathrm{m}^{3}$ during the fall and from $1.2 \times 10^{2}$ to
$9.3 \times 10^{2} \mathrm{CFU} / \mathrm{m}^{3}$ during the winter for fungi (Table 2).

Instead, inside milking parlours, in the stage after milking, therefore once the buf-

Table 1. Concentrations of airborne microrganisms measured in stables of $\mathbf{1 0}$ buffalo farms.

\begin{tabular}{|c|c|c|c|c|c|c|c|}
\hline \multirow[t]{2}{*}{ Season } & \multirow[t]{2}{*}{ Farm $\mathbf{N}^{\circ}$} & \multicolumn{3}{|c|}{ Culturable bacteria (CFU/m³) } & \multicolumn{3}{|c|}{ Culturable fungi (CFU/m³) } \\
\hline & & Range & Mean & Ds & Range & Mean & Ds \\
\hline \multicolumn{8}{|l|}{ Winter } \\
\hline & 1 & $9.3 \times 10^{4}-2.3 \times 10^{5}$ & $1.26 \times 10^{5}$ & $4.1 \times 10^{4}$ & $8.2 \times 10^{2}-1.1 \times 10^{3}$ & $9.89 \times 10^{2}$ & $0.84 \times 10^{2}$ \\
\hline & 2 & $1.5 \times 10^{5}-2.5 \times 10^{5}$ & $1.91 \times 10^{5}$ & $3.07 \times 10^{4}$ & $9,6 \times 10^{2}-1.7 \times 10^{3}$ & $1.14 \times 10^{3}$ & $2.1 \times 10^{2}$ \\
\hline & 3 & $6.3 \times 10^{3}-9.5 \times 10^{4}$ & $4.31 \times 10^{4}$ & $3.98 \times 10^{4}$ & $3.3 \times 10^{2}-4.5 \times 10^{3}$ & $3.83 \times 10^{2}$ & $0.36 \times 10^{2}$ \\
\hline & 4 & $9.4 \times 10^{4}-2.1 \times 10^{5}$ & $1.4 \times 10^{5}$ & $4.22 \times 10^{5}$ & $7.8 \times 10^{2}-9.6 \times 102$ & $8.55 \times 10^{2}$ & $0.58 \times 102$ \\
\hline & 5 & $9.8 \times 10^{3}-1.9 \times 10^{4}$ & $1.47 \times 10^{4}$ & $2.66 \times 10^{3}$ & $7.7 \times 10^{2}-8.7 \times 10^{2}$ & $8.24 \times 10^{2}$ & $0.29 \times 10^{2}$ \\
\hline & 6 & $9.8 \times 10^{4}-2.1 \times 10^{5}$ & $1.52 \times 10^{5}$ & $3.32 \times 10^{4}$ & $7.8 \times 10^{2}-1.2 \times 10^{3}$ & $8.80 \times 10^{2}$ & $1 \times 10^{2}$ \\
\hline & 7 & $2.1 \times 10^{5}-3.2 \times 10^{5}$ & $2.62 \times 10^{5}$ & $3.67 \times 10^{4}$ & $1,1 \times 10^{3}-1.7 \times 10^{3}$ & $1.3 \times 10^{2}$ & $2 \times 102$ \\
\hline & 8 & $7.5 \times 104-8.4 \times 10^{4}$ & $7.96 \times 10^{4}$ & $3,38 \times 10^{3}$ & $3.3 \times 10^{2}-4.4 \times 10^{2}$ & $3.89 \times 10^{2}$ & $0.35 \times 10^{2}$ \\
\hline & 9 & $1.2 \times 10^{5}-2.2 \times 10^{5}$ & $1.74 \times 10^{5}$ & $2.59 \times 10^{4}$ & $8.2 \times 10^{2}-9.58 \times 10^{2}$ & $8.77 \times 10^{2}$ & $0.46 \times 10^{2}$ \\
\hline & 10 & $1.1 \times 10^{5}-2,5 \times 10^{5}$ & $1.82 \times 10^{5}$ & $4.19 \times 10^{4}$ & $7.78 \times 10^{2}-9.38 \times 10$ & $8.61 \times 10^{2}$ & $0.56 \times 10^{2}$ \\
\hline \multicolumn{8}{|l|}{ Spring } \\
\hline & 1 & $6.6 \times 10^{5}-8.5 \times 10^{5}$ & $7.59 \times 10^{5}$ & $7.33 \times 10^{4}$ & $8.1 \times 10^{2}-2.4 \times 10^{3}$ & $1.65 \times 10^{3}$ & $5.32 \times 10^{2}$ \\
\hline & 2 & $8.3 \times 10^{5}-1.1 \times 106$ & $9.37 \times 10^{5}$ & $6.60 \times 10^{4}$ & $1.5 \times 10^{3}-3.3 \times 10^{3}$ & $2.46 \times 10^{3}$ & $6.25 \times 10^{2}$ \\
\hline & 3 & $5.1 \times 10^{5}-7.3 \times 10^{5}$ & $6.34 \times 10^{5}$ & $7.02 \times 10^{4}$ & $6.6 \times 10^{2}-8.9 \times 10^{2}$ & $7.69 \times 10^{2}$ & $0.75 \times 10^{2}$ \\
\hline & 4 & $6.5 \times 10^{5}-8.5 \times 10^{5}$ & $7.68 \times 10^{5}$ & $7.52 \times 10^{4}$ & $9.5 \times 10^{2}-2.4 \times 10^{3}$ & $1.73 \times 10^{3}$ & $4.97 \times 10^{2}$ \\
\hline & 5 & $6.5 \times 10^{4}-8.6 \times 10^{5}$ & $7.65 \times 10^{4}$ & $7.42 \times 10^{3}$ & $9.6 \times 10^{2}-2.7 \times 10^{3}$ & $1.72 \times 10^{3}$ & $4.93 \times 10^{2}$ \\
\hline & 6 & $6.5 \times 10^{5}-8.6 \times 10^{5}$ & $7.55 \times 105$ & $6.95 \times 10^{4}$ & $9.7 \times 10^{2}-2.3 \times 10^{3}$ & $1.70 \times 10^{3}$ & $4.46 \times 10^{2}$ \\
\hline & 7 & $8.5 \times 10^{5}-1.1 \times 10^{6}$ & $9.44 \times 10^{5}$ & $6.38 \times 10^{4}$ & $1.6 \times 10^{3}-3.3 \times 10^{3}$ & $2.49 \times 10^{3}$ & $6.11 \times 10^{2}$ \\
\hline & 8 & $4.6 \times 10^{5}-6.3 \times 10^{5}$ & $5.50 \times 10^{5}$ & $6.20 \times 10^{4}$ & $7.1 \times 10^{2}-9.1 \times 10^{2}$ & $8.26 \times 10^{2}$ & $0.72 \times 10^{2}$ \\
\hline & 9 & $6.7 \times 10^{5}-8.5 \times 10^{5}$ & $7.60 \times 10^{5}$ & $6.70 \times 10^{4}$ & $9.6 \times 10^{2}-2.6 \times 10^{2}$ & $1.74 \times 10^{3}$ & $4.74 \times 10^{2}$ \\
\hline & 10 & $6.4 \times 10^{5}-8.7 \times 10^{5}$ & $7.65 \times 10^{5}$ & $8.38 \times 10^{4}$ & $1.5 \times 10^{3}-3.3 \times 10^{3}$ & $1.69 \times 10^{3}$ & $4.23 \times 10^{2}$ \\
\hline \multicolumn{8}{|c|}{ Summer } \\
\hline & 1 & $7.6 \times 10^{5}-1.1 \times 106$ & $9.01 \times 10^{5}$ & $9.53 \times 10^{4}$ & $1.2 \times 10^{3}-2.7 \times 10^{3}$ & $1.95 \times 10^{3}$ & $6.65 \times 10^{2}$ \\
\hline & 2 & $1.2 \times 106-2.4 \times 106$ & $1.88 \times 10^{6}$ & $3.33 \times 10^{5}$ & $2.7 \times 10^{3}-4.1 \times 10^{3}$ & $3.50 \times 10^{3}$ & $4.57 \times 10^{2}$ \\
\hline & 3 & $5.6 \times 105-7.6 \times 105$ & $6.79 \times 10^{5}$ & $6.24 \times 10^{4}$ & $9.3 \times 10^{2}-1.5 \times 10^{3}$ & $1.11 \times 10^{3}$ & $1.89 \times 10^{2}$ \\
\hline & 4 & $7.8 \times 10^{5}-9.7 \times 10^{5}$ & $8.95 \times 10^{5}$ & $6.23 \times 10^{4}$ & $1.1 \times 10^{3}-3.0 \times 10^{3}$ & $2.18 \times 10^{3}$ & $4.97 \times 10^{2}$ \\
\hline & 5 & $7.9 \times 10^{4}-9.4 \times 10^{5}$ & $8.66 \times 10^{4}$ & $4.68 \times 10^{3}$ & $1.8 \times 10^{3}-2.7 \times 10^{3}$ & $2.20 \times 10^{3}$ & $2.97 \times 10^{2}$ \\
\hline & 6 & $8.6 \times 105-9.8 \times 10^{5}$ & $9.22 \times 10^{5}$ & $3.90 \times 10^{4}$ & $1.5 \times 10^{3}-2.6 \times 10^{3}$ & $2.02 \times 10^{3}$ & $3.70 \times 10^{2}$ \\
\hline & 7 & $1.3 \times 106-2.4 \times 106$ & $1.93 \times 106$ & $3.52 \times 10^{5}$ & $2.8 \times 10^{3}-4.1 \times 10^{3}$ & $3.39 \times 10^{3}$ & $4.16 \times 10^{2}$ \\
\hline & 8 & $5.7 \times 10^{5}-7.3 \times 10^{5}$ & $6.43 \times 10^{5}$ & $4.98 \times 10^{4}$ & $9.1 \times 10^{2}-1.3 \times 10^{3}$ & $1.02 \times 10^{3}$ & $1.23 \times 10^{2}$ \\
\hline & 9 & $8.4 \times 10^{5}-9.8 \times 10^{5}$ & $9.08 \times 10^{5}$ & $4.29 \times 10^{4}$ & $1.6 \times 10^{3}-2.6 \times 10^{3}$ & $2.01 \times 10^{3}$ & $3.29 \times 10^{2}$ \\
\hline & 10 & $8.5 \times 10^{5}-9.6 \times 10^{5}$ & $9.10 \times 10^{5}$ & $4.05 \times 10^{4}$ & $1.4 \times 10^{3}-2.1 \times 10^{3}$ & $2.05 \times 10^{3}$ & $4.01 \times 10^{2}$ \\
\hline \multicolumn{8}{|l|}{ Fall } \\
\hline & 1 & $6.2 \times 10^{5}-8.7 \times 10^{5}$ & $7.20 \times 10^{5}$ & $7.72 \times 10^{4}$ & $1.5 \times 10^{3}-2.2 \times 10^{3}$ & $1.74 \times 10^{3}$ & $3.63 \times 10^{2}$ \\
\hline & 2 & $8.3 \times 10^{5}-9.5 \times 10^{5}$ & $8.91 \times 10^{5}$ & $4.07 \times 10^{4}$ & $1.3 \times 10^{3}-2.9 \times 103$ & $2.13 \times 10^{3}$ & $4.86 \times 10^{2}$ \\
\hline & 3 & $5.2 \times 10^{5}-7.1 \times 10^{5}$ & $6.02 \times 10^{5}$ & $5.83 \times 10^{4}$ & $7.3 \times 10^{2}-9.1 \times 10^{2}$ & $8.22 \times 10^{2}$ & $0.55 \times 10^{2}$ \\
\hline & 4 & $6.4 \times 10^{5}-8.7 \times 10^{5}$ & $7.84 \times 10^{5}$ & $7.08 \times 10^{4}$ & $9.8 \times 10^{2}-2.2 \times 10^{3}$ & $1.69 \times 10^{3}$ & $4.06 \times 10^{2}$ \\
\hline & 5 & $6.2 \times 10^{5}-8.7 \times 10^{5}$ & $7.67 \times 10^{4}$ & $8.43 \times 10^{3}$ & $9.5 \times 10^{2}-2.3 \times 10^{3}$ & $1.64 \times 10^{3}$ & $4.50 \times 10^{2}$ \\
\hline & 6 & $6.2 \times 10^{5}-8.8 \times 10^{5}$ & $7.61 \times 10^{5}$ & $8.90 \times 10^{4}$ & $9.6 \times 10^{2}-2.4 \times 10^{3}$ & $1.69 \times 10^{3}$ & $4.84 \times 10^{2}$ \\
\hline & 7 & $8.1 \times 10^{5}-9.8 \times 10^{5}$ & $8.87 \times 10^{5}$ & $4.93 \times 10^{4}$ & $1.5 \times 10^{3}-2.9 \times 10^{3}$ & $2.22 \times 10^{3}$ & $4.79 \times 10^{2}$ \\
\hline & 8 & $4.1 \times 10^{5}-6.6 \times 10^{5}$ & $5.38 \times 10^{5}$ & $8.30 \times 10^{4}$ & $7.1 \times 10^{2}-9.0 \times 10^{2}$ & $8.0 \times 10^{2}$ & $0.60 \times 10^{2}$ \\
\hline & 9 & $6.3 \times 10^{5}-8.5 \times 10^{5}$ & $7.70 \times 10^{5}$ & $7.84 \times 10^{4}$ & $9.6 \times 10^{2}-2.3 \times 10^{3}$ & $1.67 \times 10^{3}$ & $4.66 \times 10^{2}$ \\
\hline & 10 & $6.0 \times 10^{5}-8.9 \times 10^{5}$ & 771,987 & $8.36 \times 10^{4}$ & $8.3 \times 10^{2}-1.9 \times 10^{3}$ & $1.66 \times 10^{3}$ & $4.70 \times 10^{2}$ \\
\hline
\end{tabular}

$\mathrm{CFU}$, colony-forming unit. 
falos had stationed in the rooms, the concentrations were considerably higher and ranged from $1.2 \times 10^{3}$ to $7.2 \times 10^{3} \mathrm{CFU} / \mathrm{m}^{3}$ during the spring, $9.2 \times 10^{2}$ to $8.3 \times 10^{3}$
$\mathrm{CFU} / \mathrm{m}^{3}$ during the summer, $9.2 \times 10^{2}$ to $7.6 \times 10^{3} \mathrm{CFU} / \mathrm{m}^{3}$ during the fall and $6.1 \times 10^{2}$ to $4.8 \times 10^{3} \mathrm{CFU} / \mathrm{m}^{3}$ during the winter for bacteria (Table 3) and from $6.1 \times 10^{2}$ to
$2.9 \times 10^{3} \mathrm{CFU} / \mathrm{m}^{3}$ during the spring, $7.9 \times 10^{2}$ to $3.7 \times 10^{3} \mathrm{CFU} / \mathrm{m}^{3}$ during the summer, $6.3 \times 10^{2}$ to $3.4 \times 10^{3} \mathrm{CFU} / \mathrm{m}^{3}$ during the fall and $2.6 \times 10^{2}$ to $2.0 \times 10^{3} \mathrm{CFU} / \mathrm{m}^{3}$ during the

Table 2. Concentrations of airborne microrganisms measured in stables of pre-milking of 10 buffalo farms.

\begin{tabular}{|c|c|c|c|c|c|c|c|}
\hline \multirow[t]{2}{*}{ Season } & \multirow[t]{2}{*}{ Farm $\mathbb{N}^{\circ}$} & \multicolumn{3}{|c|}{ Culturable bacteria (CFU/m³) } & \multicolumn{3}{|c|}{ Culturable fungi (CFU/m³) } \\
\hline & & Range & Mean & Ds & Range & Mean & Ds \\
\hline \multicolumn{8}{|l|}{ Winter } \\
\hline & 1 & $8.1 \times 10^{2}-1.1 \times 10^{3}$ & $9.11 \times 10^{2}$ & $0.77 \times 10^{2}$ & $3.3 \times 10^{2}-5.6 \times 10^{2}$ & $4.28 \times 10^{2}$ & $0.65 \times 10^{2}$ \\
\hline & 2 & $9.5 \times 10^{2}-1.9 \times 10^{3}$ & $1.33 \times 10^{3}$ & $3.39 \times 10^{2}$ & $7.5 \times 10^{2}-9.3 \times 10^{2}$ & $8.3 \times 10^{2}$ & $0.54 \times 10^{2}$ \\
\hline & 3 & $2.3 \times 10^{2}-2.4 \times 10^{2}$ & $2.92 \times 10^{2}$ & $0.29 \times 10^{2}$ & $1.2 \times 10^{2}-2.4 \times 10^{2}$ & $1.79 \times 10^{2}$ & $0.38 \times 10^{2}$ \\
\hline & 4 & $7.3 \times 10^{2}-1.2 \times 10^{3}$ & $8.43 \times 10^{2}$ & $1.10 \times 10^{2}$ & $3.7 \times 10^{2}-4.6 \times 10^{2}$ & $4.08 \times 10^{2}$ & $0.28 \times 10^{2}$ \\
\hline & 5 & $7.6 \times 10^{2}-8.7 \times 10^{2}$ & $8.15 \times 10^{2}$ & $0.34 \times 10^{2}$ & $3.6 \times 10^{2}-4.5 \times 10^{2}$ & $3.99 \times 10^{2}$ & $0.32 \times 10^{2}$ \\
\hline & 6 & $7.8 \times 10^{2}-9.1 \times 10^{2}$ & $8.29 \times 10^{2}$ & $0.34 \times 10^{2}$ & $4.1 \times 10^{2}-5.4 \times 10^{2}$ & $4.75 \times 10^{2}$ & $0.36 \times 10^{2}$ \\
\hline & 7 & $1 \times 10^{3}-1.7 \times 10^{3}$ & $1.37 \times 10^{3}$ & $1.86 \times 10^{2}$ & $7.3 \times 10^{2}-8.4 \times 10^{2}$ & $8 \times 10^{2}$ & $0.30 \times 10^{2}$ \\
\hline & 8 & $2.7 \times 10^{2}-4.1 \times 10^{2}$ & $3.46 \times 10^{2}$ & $0.41 \times 10^{2}$ & $1.6 \times 10^{2}-2.6 \times 10^{2}$ & $2.15 \times 10^{2}$ & $0.33 \times 10^{2}$ \\
\hline & 9 & $8.1 \times 10^{2}-9.3 \times 10^{2}$ & $8.76 \times 10^{2}$ & $0.41 \times 10^{2}$ & $4.1 \times 10^{2}-5.3 \times 10^{2}$ & $4.69 \times 10^{2}$ & $0.39 \times 102$ \\
\hline & 10 & $8.2 \times 10^{2}-9.6 \times 10^{2}$ & $8.91 \times 10^{2}$ & $0.48 \times 10^{2}$ & $3.8 \times 10^{2}-5.4 \times 10^{2}$ & $4.56 \times 10^{2}$ & $0.45 \times 10^{2}$ \\
\hline \multicolumn{8}{|l|}{ Spring } \\
\hline & 1 & $1.1 \times 10^{3}-2.6 \times 10^{3}$ & $1.77 \times 10^{3}$ & $1.06 \times 10^{3}$ & $6.2 \times 10^{2}-9.7 \times 10^{2}$ & $8.04 \times 10^{2}$ & $0.65 \times 10^{2}$ \\
\hline & 2 & $1.3 \times 10^{3}-2.9 \times 10^{3}$ & $2.17 \times 10^{3}$ & $1.13 \times 10^{3}$ & $8.2 \times 10^{2}-1.1 \times 10^{3}$ & $9.26 \times 10^{2}$ & $0.54 \times 10^{2}$ \\
\hline & 3 & $7.1 \times 10^{2}-9.1 \times 10^{2}$ & $8.23 \times 10^{2}$ & $1.41 \times 10^{2}$ & $2.3 \times 10^{2}-4.2 \times 10^{2}$ & $3.26 \times 10^{2}$ & $0.38 \times 10^{2}$ \\
\hline & 4 & $9.4 \times 10^{2}-2.6 \times 10^{3}$ & $1.71 \times 10^{3}$ & $1.17 \times 10^{3}$ & $6.1 \times 10^{2}-9.7 \times 10^{2}$ & $8.03 \times 10^{2}$ & $0.28 \times 10^{2}$ \\
\hline & 5 & $9.5 \times 10^{2}-2.6 \times 10^{3}$ & $1.70 \times 10^{3}$ & $1.17 \times 10^{3}$ & $6.2 \times 10^{2}-9.7 \times 10^{2}$ & $7.87 \times 10^{2}$ & $0.32 \times 10^{2}$ \\
\hline & 6 & $1.3 \times 10^{3}-2.6 \times 10^{3}$ & $1.82 \times 10^{3}$ & $9.19 \times 10^{2}$ & $6.3 \times 10^{2}-9.5 \times 10^{2}$ & $8.09 \times 10^{2}$ & $0.36 \times 10^{2}$ \\
\hline & 7 & $1.5 \times 10^{3}-2.9 \times 10^{3}$ & $2.14 \times 10^{3}$ & $9.90 \times 10^{2}$ & $8 \times 10^{2}-1.1 \times 10^{3}$ & $9.29 \times 10^{2}$ & $0.30 \times 10^{2}$ \\
\hline & 8 & $7.2 \times 10^{2}-9.4 \times 10^{2}$ & $8.24 \times 10^{2}$ & $1.56 \times 10^{2}$ & $3.3 \times 10^{2}-4.6 \times 10^{2}$ & $3.92 \times 10^{2}$ & $0.33 \times 10^{2}$ \\
\hline & 9 & $9.4 \times 10^{2}-2.8 \times 10^{3}$ & $1.72 \times 10^{3}$ & $1.31 \times 10^{3}$ & $6.3 \times 10^{2}-9.6 \times 10^{2}$ & $8.04 \times 10^{2}$ & $0.39 \times 10^{2}$ \\
\hline & 10 & $1.1 \times 10^{3}-2.7 \times 10^{3}$ & $1.82 \times 10^{3}$ & $1.13 \times 10^{3}$ & $6.5 \times 10^{2}-9.6 \times 10^{2}$ & $8.06 \times 10^{2}$ & $0.45 \times 10^{2}$ \\
\hline \multicolumn{8}{|c|}{ Summer } \\
\hline & 1 & $1.3 \times 10^{3}-2.6 \times 10^{3}$ & $1.88 \times 10^{3}$ & $3.77 \times 10^{2}$ & $7.3 \times 10^{2}-9.7 \times 10^{2}$ & $8.86 \times 10^{2}$ & $0.63 \times 10^{2}$ \\
\hline & 2 & $3.3 \times 10^{3}-4.4 \times 10^{3}$ & $3.85 \times 10^{3}$ & $3.25 \times 10^{2}$ & $9.4 \times 10^{2}-2.2 \times 10^{3}$ & $1.67 \times 10^{3}$ & $4.1 \times 10^{2}$ \\
\hline & 3 & $9.2 \times 10^{2}-1.3 \times 10^{3}$ & $1.02 \times 10^{3}$ & $1.19 \times 10^{2}$ & $3.1 \times 10^{2}-4.7 \times 10^{2}$ & $3.93 \times 10^{2}$ & $0.51 \times 10^{2}$ \\
\hline & 4 & $1.6 \times 10^{3}-2.3 \times 10^{3}$ & $1.95 \times 10^{3}$ & $2.53 \times 10^{2}$ & $7.9 \times 10^{2}-9.7 \times 10^{2}$ & $8.89 \times 10^{2}$ & $0.60 \times 10^{2}$ \\
\hline & 5 & $1.7 \times 10^{3}-2.6 \times 10^{3}$ & $2.15 \times 10^{3}$ & $2.47 \times 10^{2}$ & $3.4 \times 10^{2}-9.4 \times 10^{2}$ & $8.33 \times 10^{2}$ & $1.46 \times 10^{2}$ \\
\hline & 6 & $1.4 \times 10^{3}-2.3 \times 10^{3}$ & $1.83 \times 10^{3}$ & $2.72 \times 10^{2}$ & $8.2 \times 10^{2}-9.4 \times 10^{2}$ & $8.79 \times 10^{2}$ & $0.36 \times 10^{2}$ \\
\hline & 7 & $3.1 \times 10^{3}-4.1 \times 10^{3}$ & $3.62 \times 10^{3}$ & $3.07 \times 10^{2}$ & $9.8 \times 10^{2}-2.0 \times 10^{3}$ & $1.41 \times 10^{3}$ & $3.15 \times 10^{2}$ \\
\hline & 8 & $9.1 \times 10^{2}-1.2 \times 10^{3}$ & $9.92 \times 10^{2}$ & $0.84 \times 10^{2}$ & $3.7 \times 10^{2}-5.3 \times 10^{2}$ & $4.57 \times 10^{2}$ & $0.48 \times 10^{2}$ \\
\hline & 9 & $1.4 \times 10^{3}-2.4 \times 10^{3}$ & $1.86 \times 10^{3}$ & $3.27 \times 10^{2}$ & $8.3 \times 10^{2}-9.7 \times 10^{2}$ & $8.99 \times 10^{2}$ & $0.43 \times 10^{2}$ \\
\hline & 10 & $1.5 \times 10^{3}-2.5 \times 10^{3}$ & $2.10 \times 10^{3}$ & $2.98 \times 10^{2}$ & $8.1 \times 10^{2}-9.6 \times 10^{2}$ & $8.88 \times 10^{2}$ & $0.43 \times 10^{2}$ \\
\hline \multicolumn{8}{|l|}{ Fall } \\
\hline & 1 & $9.3 \times 10^{2}-2.3 \times 10^{3}$ & $1.61 \times 10^{3}$ & $4.65 \times 10^{2}$ & $6.3 \times 10^{2}-8.4 \times 10^{2}$ & $7.61 \times 10^{2}$ & $0.66 \times 10^{2}$ \\
\hline & 2 & $1.2 \times 10^{3}-3.2 \times 10^{3}$ & $2.25 \times 10^{3}$ & $6.25 \times 10^{2}$ & $7.9 \times 10^{2}-9.9 \times 10^{2}$ & $9.13 \times 10^{2}$ & $0.59 \times 10^{2}$ \\
\hline & 3 & $6.1 \times 10^{2}-9.2 \times 10^{2}$ & $7.25 \times 10^{2}$ & $0.84 \times 10^{2}$ & $2.6 \times 10^{2}-4.0 \times 10^{2}$ & $3.36 \times 10^{2}$ & $0.48 \times 10^{2}$ \\
\hline & 4 & $9.3 \times 10^{2}-2.3 \times 10^{3}$ & $1.66 \times 10^{3}$ & $4.82 \times 10^{2}$ & $6.3 \times 10^{2}-8.4 \times 10^{2}$ & $7.59 \times 10^{2}$ & $0.66 \times 10^{2}$ \\
\hline & 5 & $9.1 \times 10^{2}-2.3 \times 10^{3}$ & $1.62 \times 10^{3}$ & $4.69 \times 10^{2}$ & $6.3 \times 10^{2}-8.5 \times 10^{2}$ & $7.6 \times 10^{2}$ & $0.65 \times 10^{2}$ \\
\hline & 6 & $8.3 \times 10^{2}-2.2 \times 10^{3}$ & $1.50 \times 10^{3}$ & $4.46 \times 10^{2}$ & $6.8 \times 10^{2}-8.6 \times 10^{2}$ & $7.68 \times 10^{2}$ & $0.74 \times 10^{2}$ \\
\hline & 7 & $1.1 \times 10^{3}-3.2 \times 10^{3}$ & $2.19 \times 10^{3}$ & $6.98 \times 10^{2}$ & $7.8 \times 10^{2}-9.9 \times 10^{2}$ & $9.02 \times 10^{2}$ & $0.67 \times 10^{2}$ \\
\hline & 8 & $6.4 \times 10^{2}-8.2 \times 10^{2}$ & $7.44 \times 10^{2}$ & $0.60 \times 10^{2}$ & $3.2 \times 10^{2}-4.3 \times 10^{2}$ & $3.79 \times 10^{2}$ & $0.37 \times 10^{2}$ \\
\hline & 9 & $9.9 \times 10^{2}-2.3 \times 10^{3}$ & $1.73 \times 10^{3}$ & $3.86 \times 10^{2}$ & $6.4 \times 10^{2}-8.6 \times 10^{2}$ & $7.64 \times 10^{2}$ & $0.70 \times 10^{2}$ \\
\hline & 10 & $9.3 \times 10^{2}-2.4 \times 10^{3}$ & $1.63 \times 10^{3}$ & $4.82 \times 10^{2}$ & $6.5 \times 10^{2}-8.5 \times 10^{2}$ & $7.64 \times 10^{2}$ & $0.66 \times 10^{2}$ \\
\hline
\end{tabular}

CFU, colony-forming unit. 
winter for fungi (Table 3). For an immediate understanding the results are show in a histogram in Figure 3 for the mean value of bacteria concentrations and in a histogram in Figure 4 for the mean value of fungi concentrations.

Many differences have been found as regard the level of bacteria and fungi observed between the different stables during the examined period: in particular two farms have constantly scored low levels of contamination while two different farms

Table 3. Concentrations of airborne microrganisms measured in stables of post-milking bacteria of 10 buffalo farms.

\begin{tabular}{|c|c|c|c|c|c|c|c|}
\hline \multirow[t]{2}{*}{ Season } & \multirow[t]{2}{*}{ Farm $\mathbf{N}^{\circ}$} & \multicolumn{3}{|c|}{ Culturable bacteria (CFU/m³) } & \multicolumn{3}{|c|}{ Culturable fungi (CFU/m³) } \\
\hline & & Range & Mean & Ds & Range & Mean & Ds \\
\hline \multicolumn{8}{|l|}{ Winter } \\
\hline & 1 & $1.7 \times 103-2.6 \times 10^{3}$ & $2.15 \times 10^{3}$ & $2.77 \times 10^{2}$ & $7.3 \times 10^{2}-1.0 \times 10^{3}$ & $8.19 \times 10^{2}$ & $0.70 \times 10^{2}$ \\
\hline & 2 & $2.9 \times 10^{3}-4.8 \times 10^{3}$ & $3.77 \times 10^{3}$ & $6.17 \times 10^{2}$ & $9.6 \times 10^{2}-2.0 \times 10^{3}$ & $1.35 \times 10^{3}$ & $4.08 \times 10^{2}$ \\
\hline & 3 & $6.1 \times 10^{2}-9.5 \times 10^{2}$ & $7.24 \times 10^{2}$ & $0.94 \times 10^{2}$ & $2.7 \times 10^{2}-2.8 \times 10^{2}$ & $3.28 \times 10^{2}$ & $0.31 \times 10^{2}$ \\
\hline & 4 & $1.7 \times 10^{3}-2.4 \times 10^{3}$ & $2.04 \times 10^{3}$ & $2.47 \times 10^{2}$ & $7.3 \times 10^{2}-8.4 \times 10^{2}$ & $7.75 \times 10^{2}$ & $0.34 \times 10^{2}$ \\
\hline & 5 & $1.3 \times 10^{3}-2.4 \times 10^{3}$ & $1.84 \times 10^{3}$ & $3.34 \times 10^{2}$ & $6.3 \times 10^{2}-8.2 \times 10^{2}$ & $7.29 \times 10^{2}$ & $0.54 \times 10^{2}$ \\
\hline & 6 & $1.6 \times 10^{3}-2.5 \times 10^{3}$ & $1.99 \times 10^{3}$ & $2.77 \times 10^{2}$ & $7.4 \times 10^{2}-8.6 \times 10^{2}$ & $8.02 \times 10^{2}$ & $0.38 \times 10^{2}$ \\
\hline & 7 & $2.9 \times 10^{3}-3.8 \times 10^{3}$ & $3.27 \times 10^{3}$ & $2.73 \times 10^{2}$ & $1.0 \times 10^{3}-1.6 \times 10^{3}$ & $1.29 \times 10^{3}$ & $1.90 \times 10^{2}$ \\
\hline & 8 & $7.9 \times 10^{2}-8.7 \times 10^{2}$ & $8.31 \times 10^{2}$ & $0.25 \times 10^{2}$ & $2.6 \times 10^{2}-3.9 \times 10^{2}$ & $3.36 \times 10^{2}$ & $0.43 \times 10^{2}$ \\
\hline & 9 & $1.1 \times 10^{3}-2.2 \times 10^{3}$ & $1.56 \times 10^{3}$ & $3.37 \times 10^{2}$ & $7.7 \times 10^{2}-9.2 \times 10^{2}$ & $8.46 \times 10^{2}$ & $0.54 \times 10^{2}$ \\
\hline & 10 & $1.2 \times 10^{3}-2.3 \times 10^{3}$ & $1.69 \times 10^{3}$ & $3.57 \times 10^{2}$ & $7.2 \times 10^{2}-9.2 \times 10^{2}$ & $7.96 \times 10^{2}$ & $0.52 \times 10^{2}$ \\
\hline \multicolumn{8}{|l|}{ Spring } \\
\hline & 1 & $3.7 \times 10^{3}-4.6 \times 10^{3}$ & $4.09 \times 10^{3}$ & $6.36 \times 10^{2}$ & $9.5 \times 10^{2}-1.8 \times 10^{3}$ & $1.23 \times 10^{3}$ & $3.44 \times 10^{2}$ \\
\hline & 2 & $5.3 \times 10^{3}-7.2 \times 10^{3}$ & $6.28 \times 10^{3}$ & $1.34 \times 10^{3}$ & $1.3 \times 10^{3}-2.9 \times 10^{3}$ & $2.35 \times 10^{3}$ & $5.05 \times 10^{2}$ \\
\hline & 3 & $3.4 \times 10^{3}-4.6 \times 10^{3}$ & $4.07 \times 10^{3}$ & $8.48 \times 10^{2}$ & $6.1 \times 10^{2}-9.2 \times 10^{2}$ & $7.46 \times 10^{2}$ & $0.95 \times 10^{2}$ \\
\hline & 4 & $3.4 \times 10^{3}-4.6 \times 10^{3}$ & $4.06 \times 10^{3}$ & $8.48 \times 10^{3}$ & $9.5 \times 10^{2}-1.9 \times 10^{3}$ & $1.25 \times 10^{3}$ & $3.45 \times 10^{2}$ \\
\hline & 5 & $3.4 \times 10^{3}-4.6 \times 10^{3}$ & $4.06 \times 10^{3}$ & $8.48 \times 10^{2}$ & $9.5 \times 10^{2}-1.9 \times 10^{3}$ & $1.24 \times 10^{3}$ & $3.21 \times 10^{2}$ \\
\hline & 6 & $3.5 \times 10^{3}-4.7 \times 10^{3}$ & $4.04 \times 10^{3}$ & $8.48 \times 10^{2}$ & $9.5 \times 10^{2}-1.9 \times 10^{3}$ & $1.17 \times 10^{3}$ & $2.94 \times 10^{2}$ \\
\hline & 7 & $5.3 \times 10^{3}-7.1 \times 10^{3}$ & $6.39 \times 10^{3}$ & $1.27 \times 10^{3}$ & $1.5 \times 10^{3}-2.9 \times 10^{3}$ & $2.32 \times 10^{3}$ & $5.20 \times 10^{2}$ \\
\hline & 8 & $1.2 \times 10^{3}-2.8 \times 10^{3}$ & $1.91 \times 10^{3}$ & $1.13 \times 10^{3}$ & $7.1 \times 10^{2}-9.2 \times 10^{2}$ & $8.19 \times 10^{2}$ & $0.61 \times 10^{2}$ \\
\hline & 9 & $3.5 \times 10^{3}-4.7 \times 10^{3}$ & $4.08 \times 10^{3}$ & $8.48 \times 10^{2}$ & $9.4 \times 10^{2}-1.9 \times 10^{3}$ & $1.26 \times 10^{3}$ & $3.41 \times 10^{2}$ \\
\hline & 10 & $3.6 \times 10^{3}-4.7 \times 10^{3}$ & $4.07 \times 10^{3}$ & $7.78 \times 10^{2}$ & $9.4 \times 10^{2}-1.9 \times 10^{3}$ & $1.33 \times 10^{3}$ & $3.66 \times 10^{2}$ \\
\hline \multicolumn{8}{|c|}{ Summer } \\
\hline & 1 & $4.2 \times 10^{3}-5.5 \times 10^{3}$ & $4.96 \times 10^{3}$ & $3.77 \times 10^{2}$ & $1.1 \times 10^{3}-2.0 \times 10^{3}$ & $1.61 \times 10^{3}$ & $2.63 \times 10^{2}$ \\
\hline & 2 & $6.9 \times 10^{3}-8.1 \times 10^{3}$ & $7.51 \times 10^{3}$ & $3.80 \times 10^{2}$ & $2.6 \times 10^{3}-3.7 \times 10^{3}$ & $3.06 \times 10^{3}$ & $3.54 \times 10^{2}$ \\
\hline & 3 & $9.2 \times 10^{2}-1.2 \times 10^{3}$ & $1.41 \times 10^{3}$ & $2.82 \times 10^{2}$ & $7.9 \times 10^{2}-9.9 \times 10^{2}$ & $8.93 \times 10^{2}$ & $0.60 \times 10^{2}$ \\
\hline & 4 & $4.4 \times 10^{3}-5.5 \times 10^{3}$ & $5.01 \times 10^{3}$ & $3.33 \times 10^{2}$ & $1.3 \times 10^{3}-2.3 \times 10^{3}$ & $1.81 \times 10^{3}$ & $3.16 \times 10^{2}$ \\
\hline & 5 & $4.3 \times 10^{3}-5.4 \times 10^{3}$ & $4.92 \times 10^{3}$ & $3.49 \times 10^{2}$ & $1.3 \times 10^{3}-2.2 \times 10^{3}$ & $1.77 \times 10^{3}$ & $2.55 \times 10^{2}$ \\
\hline & 6 & $4.4 \times 10^{3}-5.6 \times 10^{3}$ & $4.95 \times 10^{3}$ & $3.41 \times 10^{2}$ & $1.2 \times 10^{3}-2.2 \times 10^{3}$ & $1.71 \times 10^{3}$ & $3.03 \times 10^{2}$ \\
\hline & 7 & $6.7 \times 10^{3}-8.3 \times 10^{3}$ & $7.47 \times 10^{3}$ & $4.75 \times 10^{2}$ & $1.2 \times 10^{3}-3.6 \times 10^{3}$ & $3.02 \times 10^{3}$ & $5.83 \times 10^{2}$ \\
\hline & 8 & $1.9 \times 10^{3}-2.8 \times 10^{3}$ & $2.36 \times 10^{3}$ & $2.95 \times 10^{2}$ & $8.3 \times 10^{2}-9.6 \times 10^{2}$ & $9.01 \times 10^{2}$ & $0.39 \times 10^{2}$ \\
\hline & 9 & $4.2 \times 10^{3}-5.6 \times 10^{3}$ & $4.85 \times 10^{3}$ & $4.43 \times 10^{2}$ & $9.8 \times 10^{2}-2.1 \times 10^{3}$ & $1.53 \times 10^{3}$ & $3.45 \times 10^{2}$ \\
\hline & 10 & $4.1 \times 10^{3}-5.4 \times 10^{3}$ & $4.89 \times 10^{3}$ & $3.99 \times 10^{2}$ & $1.1 \times 10^{3}-2.1 \times 10^{3}$ & $1.58 \times 10^{3}$ & $2.89 \times 10^{2}$ \\
\hline \multicolumn{8}{|l|}{ Fall } \\
\hline & 1 & $2.9 \times 10^{3}-4.3 \times 10^{3}$ & $3.79 \times 10^{3}$ & $4.29 \times 10^{2}$ & $9.3 \times 10^{2}-1.7 \times 10^{3}$ & $1.22 \times 10^{3}$ & $2.69 \times 10^{2}$ \\
\hline & 2 & $5.2 \times 10^{3}-7.2 \times 10^{3}$ & $6.26 \times 10^{3}$ & $6.49 \times 10^{2}$ & $1.7 \times 10^{3}-3.2 \times 10^{3}$ & $2.28 \times 10^{3}$ & $4.72 \times 10^{2}$ \\
\hline & 3 & $9.2 \times 10^{2}-2.1 \times 10^{3}$ & $1.41 \times 10^{3}$ & $3.82 \times 10^{2}$ & $6.3 \times 10^{2}-9.2 \times 10^{2}$ & $7.65 \times 10^{2}$ & $0.86 \times 10^{2}$ \\
\hline & 4 & $2.6 \times 10^{3}-4.3 \times 10^{3}$ & $3.76 \times 10^{3}$ & $4.88 \times 10^{2}$ & $9.1 \times 10^{2}-1.7 \times 10^{3}$ & $1.25 \times 10^{3}$ & $2.88 \times 10^{2}$ \\
\hline & 5 & $2.8 \times 10^{3}-4.3 \times 10^{3}$ & $3.75 \times 10^{3}$ & $4.56 \times 10^{2}$ & $8.1 \times 10^{2}-1.7 \times 10^{3}$ & $1.18 \times 10^{3}$ & $2.46 \times 10^{2}$ \\
\hline & 6 & $2.9 \times 10^{3}-4.5 \times 10^{3}$ & $3.77 \times 10^{3}$ & $4.68 \times 10^{2}$ & $9.6 \times 10^{2}-1.6 \times 10^{3}$ & $1.22 \times 10^{3}$ & $2.39 \times 10^{2}$ \\
\hline & 7 & $5.4 \times 10^{3}-7.6 \times 10^{3}$ & $6.48 \times 10^{3}$ & $6.57 \times 10^{2}$ & $1.5 \times 10^{3}-3.4 \times 10^{3}$ & $2.30 \times 10^{3}$ & $5.64 \times 10^{2}$ \\
\hline & 8 & $1.3 \times 10^{3}-2.2 \times 10^{3}$ & $1.71 \times 10^{3}$ & $2.74 \times 10^{2}$ & $6.9 \times 10^{2}-8.5 \times 10^{2}$ & $7.80 \times 10^{2}$ & $0.58 \times 10^{2}$ \\
\hline & 9 & $2.7 \times 10^{3}-4.3 \times 10^{3}$ & $3.77 \times 10^{3}$ & $5.23 \times 10^{2}$ & $9.2 \times 10^{2}-1.8 \times 10^{3}$ & $1.23 \times 10^{3}$ & $2.80 \times 10^{2}$ \\
\hline & 10 & $2.9 \times 10^{3}-4.4 \times 10^{3}$ & $3.80 \times 10^{3}$ & $4.76 \times 10^{2}$ & $9.7 \times 10^{2}-1.5 \times 10^{3}$ & $1.30 \times 10^{3}$ & $1.96 \times 10^{2}$ \\
\hline
\end{tabular}

$\mathrm{CFU}$, colony-forming unit. 
have been constantly yielding high numbers of microorganisms (data not shown). The highest concentration was found in the stables number 2 and 7, which showed a significantly statistical prevalence compared to airborne levels of bacteria and fungi determined in the other stables. The data reported are clear, so it is very important to recommend the dirty ones to improve on the cleaning practices that should be performed more in depth and with higher frequency.

In our study, the highest concentrations of microorganisms have always been found in stables where adult animals are kept, while the lowest concentrations have been found in milking rooms, especially in the stage before milking.

The values reported for samplings performed during the post-milking stage were considerably high so it is advisable for all practitioners and workers in the farms to carry out an accurate cleaning during this phase. As regard to atmospheric conditions, on windy days different microbial concentrations were obtained. We found that on windy and wet days, the total flora levels tended to decline, whereas on windy and dry days numbers of microorganisms reached higher levels. Moreover, the amounts of airborne microorganisms changed according to the animals' diet, indeed in stables hosting adult animals fed with corn the Gram-negative bacteria concentration was significantly higher compared to those farms where animals are fed with hay. Furthermore, in the same farms the yeast concentration was significantly higher.

\section{Discussion}

This study was designed to obtain information about the airborne microorganisms present in buffalo farms of Southern Italy. No bacteria were considered to be pathogenic to the bovine respiratory system and all fungi discovered were also considered to be non-pathogenic..$^{20,21}$ However, these techniques were frequently used in bioaerosol field studies. ${ }^{22,23} \mathrm{Gas}$ as $\mathrm{NH}_{3}$ and $\mathrm{CO}_{2}$ and vapors can influence the vitality of bacteria and fungi and it is likely that some Gram negative bacteria were in a viable but not in a cultivable state. ${ }^{24}$

The mean concentrations of bacteria and fungi present in stables were conspicuous respect to concentration reported for the milking parlours, especially during the stage before milking. ${ }^{25,26}$ The cleaning of milking parlours by water spraying was performed routinely before milking and this practice probably decreased the concentrations of airborne microorganisms obtained

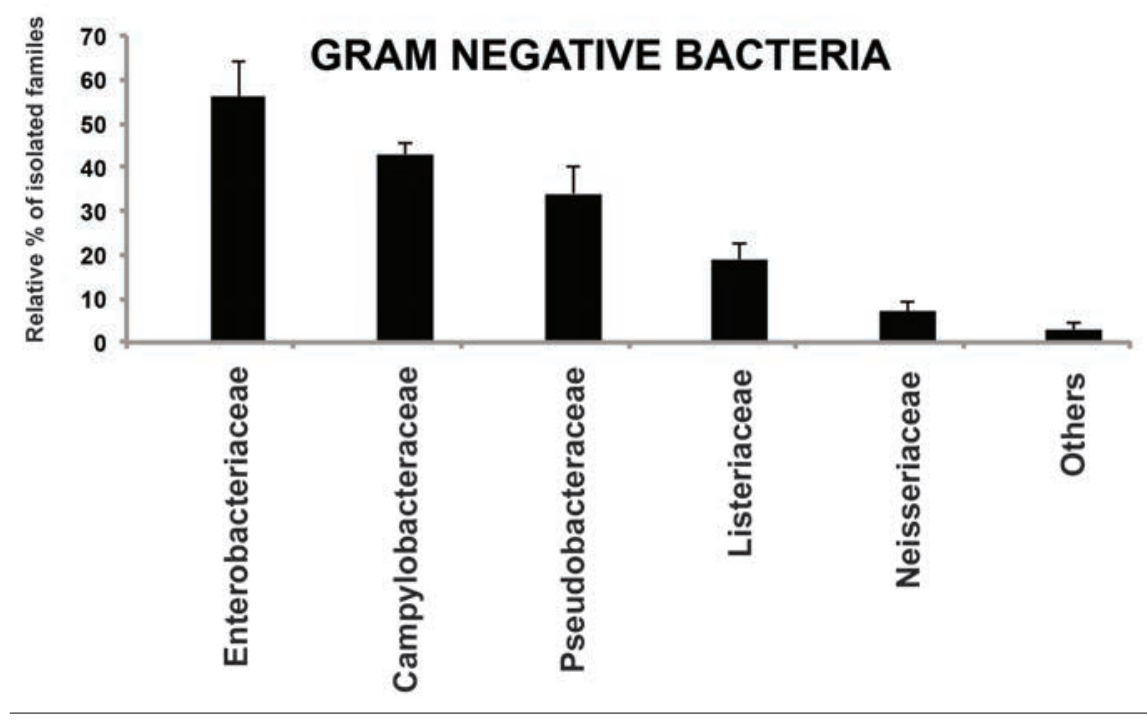

Figure 2. Gram negative bacteria founded in all areas and locations of the ten farms analyzed.

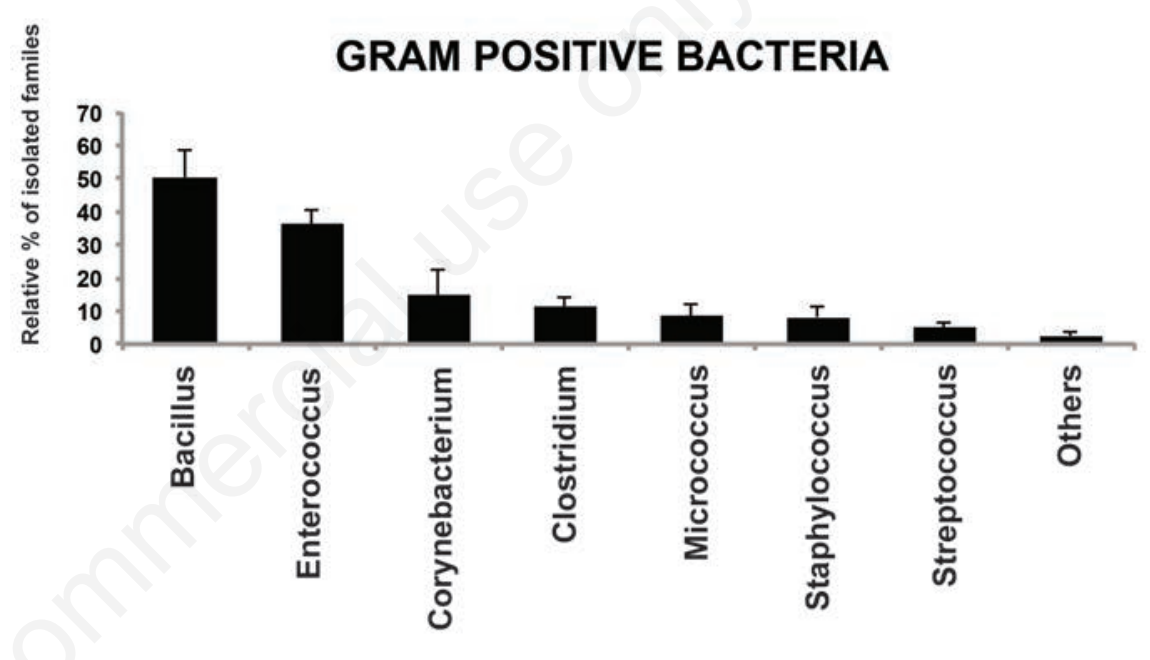

Figure 3. Gram positive bacteria founded in all areas and locations of the ten farms analyzed.

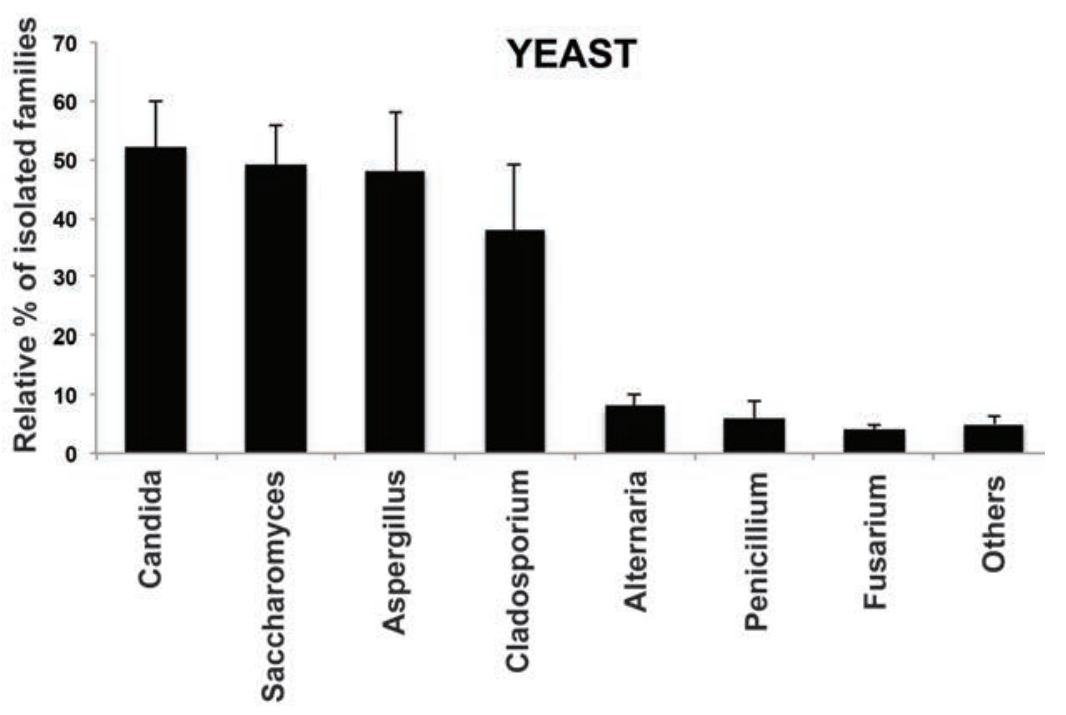

Figure 4. Yeast founded in all areas and locations of the ten farms analyzed. 
in milking parlours, increasing the difference between the two stage of milking and between the milking parlour and the stables. ${ }^{14}$ So, thorough cleaning is strongly recommended during the post milking phase. Our finding suggests that a scrupulous control of sanitation of housing, equipment and staff had an important role in minimizing the impact of the number of microorganisms presented in stables and on the hygienic quality of air. Air concentrations of microorganisms were not significantly affected by natural ventilation regimen. Species of airborne bacteria collected in the present investigation have been identified in a follow-up study, in which Micrococcus and Staphylococcus spp. were identified as predominant. Generally, only small quantities of culturable airborne Gram-negative bacteria were isolated in each of the animal houses. ${ }^{27}$ The environmental factors affecting the stables may also play a role in microbial recovery of viable and cultivable microorganisms. The predominant genera were Enterobacteriaceae, Pseudomonadaceae and Neisseriaceae. The concentration of airborne Pseudomonadaceae seems to directly related to our humidity. The airborne concentration of Enterobacteriaceae in stables is likely to increase during handling of manure and excrements compared to milking parlours. Fungal concentrations found in this study were generally lower than the concentration of bacteria. Cladosporium and Aspergillus were always the predominant fungi in the buffalo farms and among those identified in the present study Aspergillus and Penicillium are generally considered common allergens.

The high level of airborne bacteria and the presence of potential dangerous fungal genera implied the prospect of health hazards for animals, workers and staff. ${ }^{28}$ As a matter of fact, different components of cell wall of bacteria and fungi can exert a toxic effect and can be allergenic. ${ }^{29}$ Lipopolysaccharides (LPS) from Gram negative bacteria can be toxic and its effects are known to persist for long periods (about for $26 \mathrm{~h}$ ), therefore, it is possible that the effect of LPS together with the irritant properties of dust could predispose buffalo and also operators and workers to respiratory healthrelated problems. ${ }^{30}$

A further element enhancing the formation of aerosol is represented by the movement by agricultural machines and animals, which allows the persistence of microorganisms in environmental air. Even the diet to which animals are subjected plays a significant role in the air composition of the stables. No significant changes were observed in the concentration of bacteria and prevalent genera. Yet, as far as fungi are concerned, a higher concentration was observed in the air of stables where animals were fed with a mostly corn-based diet. In stables where animals were mainly fed with hay, however, an increase of the Fusarium and Alternaria species was observed.

\section{Conclusions}

In conclusion, the results show that in stables where animals and workers are in good health, no airborne aerosol particles containing pathogenic microorganisms are present.

The air however presents some microorganisms that can, due to their components (for example LPS) be potentially harmful for animals and staff working in the stables, as these items may induce inflammatory reactions in the mucous membranes of exposed subjects. Continuous stimulation and inflammation of respiratory tract mucous membranes could prepare the way for a rapid invasion by pathogenic organisms. It would be strongly advised and useful to reduce as much as possible, the concentration microorganisms from the air in farm stables, even if these are not pathogenic.

\section{References}

1. Lugauskas A, Krikstaponis A, Sveistyte L. Airborne fungi in industrial environments-potential agents of respiratory diseases. Ann Agric Environ Med 2004;11:19-25.

2. de la Cruz-Cruz LA, GuerreroLegarreta I, Ramirez R, et al. The behaviour and productivity of water buffalo in different breeding systems: a review. Vet Med 2014;59:181-93.

3. Andersen AA. New sampler for the collection, sizing, and enumeration of viable airborne particles. J Bacteriol 1958;76:471-84.

4. Durakli Velioglu S, Ercioglu E, Boyaci IH. Rapid discrimination between buffalo and cow milk and detection of adulteration of buffalo milk with cow milk using synchronous fluorescence spectroscopy in combination with multivariate methods. J Dairy Res 2017;84: 214-9.

5. Crook B, Robertson JF, Glass SA, et al. Airborne dust, ammonia, microorganisms, and antigens in pig confinement houses and the respiratory health of exposed farm workers. Am Ind Hyg Assoc J 1991;52:271-9.

6. Skora J, Matusiak K, Wojewodzki P, et al. Evaluation of microbiological and chemical contaminants in poultry farms. Int J Environ Res Public Health 2016;13:192.

7. Dutkiewicz J, Pomorski ZJH, Sitkowska J, et al. Airborne microorganisms and endotoxin in animal houses. Grana 1994;33:85-90.

8. Blaak H, van Hoek AH, Hamidjaja RA, et al. Distribution, numbers, and diversity of ESBL-producing E. coli in the poultry farm environment. PLoS One 2015;10:e0135402.

9. Radon K, Danuser B, Iversen M, et al. Air contaminants in different European farming environments. Ann Agric Environ Med 2002;9:41-8.

10. Fujiyoshi S, Tanaka D, Maruyama F. Transmission of airborne bacteria across built environments and its measurement standards: a review. Front Microbiol 2017;8:2336.

11. Gorny RL, Dutkiewicz J. Bacterial and fungal aerosols in indoor environment in Central and Eastern European countries. Ann Agric Environ Med 2002;9:17-23.

12. Chang CW, Chung H, Huang CF, Su HJ. Exposure of workers to airborne microorganisms in open-air swine houses. Appl Environ Microbiol 2001;67:155-61.

13. Matkovic K, Vucemilo M, Vinkovic B. Airborne fungi in dwellings for dairy cows and laying hens. Arh Hig Rada Toksikol 2009;60:395-9.

14. Duchaine C, Grimard Y, Cormier Y. Influence of building maintenance, environmental factors, and seasons on airborne contaminants of swine confinement buildings. AIHAJ 2000;61:5663.

15. Braun-Fahrlander C, Riedler J, Herz U, et al. Environmental exposure to endotoxin and its relation to asthma in school-age children. $\mathrm{N}$ Engl J Med 2002;347:869-77.

16. Poole JA. Farming-associated environmental exposures and effect on atopic diseases. Ann Allergy Asthma Immunol 2012;109:93-8.

17. Patbandha TK, Marandi S, Ravikala K, et al. Association of milk components with intra-mammary inflammation in Jaffrabadi buffaloes. Vet World 2015;8:989-93.

18. Little DA, Minson DJ. Variation in the phosphorus content of bone samples obtained from the last three ribs of cattle. Res Vet Sci 1977;23:393-4.

19. Barnett HL, Hunter BB. Illustrated genera of imperfect fungi. 4th ed. St. Paul: APS Press; 1998.

20. Roque K, Lim GD, Jo JH, et al. 
Epizootiological characteristics of viable bacteria and fungi in indoor air from porcine, chicken, or bovine husbandry confinement buildings. J Vet Sci 2016;17:531-8.

21. Polymenakou PN, Mandalakis M, Stephanou EG, Tselepides A. Particle size distribution of airborne microorganisms and pathogens during an Intense African dust event in the eastern Mediterranean. Environ Health Perspect 2008;116:292-6.

22. Srikanth P, Sudharsanam S, Steinberg R. Bio-aerosols in indoor environment: composition, health effects and analysis. Indian J Med Microbiol 2008;26:302-12.

23. Frac M, Hannula SE, Belka M,
Jedryczka M. Fungal biodiversity and their role in soil health. Front Microbiol 2018;9:707.

24. Attwood P, Brouwer R, Ruigewaard P, et al. A study of the relationship between airborne contaminants and environmental factors in Dutch swine confinement buildings. Am Ind Hyg Assoc J 1987;48:745-51.

25. St-Germain G, Summerbell R. Identifying filamentous fungi. A clinical laboratory handbook. Belmont: Star Publishing; 2003.

26. Yao HQ, Choi HL, Lee JH, et al. Effect of microclimate on particulate matter, airborne bacteria, and odorous compounds in swine nursery houses. J Anim Sci 2010;88:3707-14.
27. Gleeson D, O'Brien B, Flynn J, et al. Effect of pre-milking teat preparation procedures on the microbial count on teats prior to cluster application. Ir Vet J 2009;62:461-7.

28. Oliver SP, Jayarao BM, Almeida RA. Foodborne pathogens in milk and the dairy farm environment: food safety and public health implications. Foodborne Pathog Dis 2005;2:115-29.

29. Arana DM, Prieto D, Roman E, et al. The role of the cell wall in fungal pathogenesis. Microb Biotechnol 2009;2:308-20.

30. Wang X, Quinn PJ. Endotoxins: lipopolysaccharides of gram-negative bacteria. Subcell Biochem 2010;53:325. 